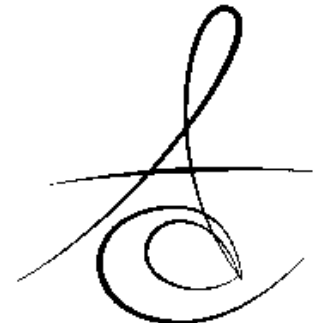

Makale Kodu/Article code: 1214

Makale Gönderilme tarihi: 18.06 .2013

Kabul Tarihi: 23.10.2013

\section{İKİ FARKLI KANAL PATININ FARKLI İRRİGASYON SOLÜSYONLARI KULLANILARAK PUSH-OUT YÖNTEMİ İLE DENTİNE BAĞLANMA DİRENÇLERİNİN DEĞERLENDİRİLMESİ}

\section{EVALUATION OF TWO DIFFERENT ROOT CANAL SEALER, USING DIFFERENT IRRIGATION SOLUTION ON PUSH OUT BOND STRENGHT TO DENTIN}

\author{
Dr. Ersan ÇiçEK* Dr. Neslihan Büşra ÖZEROL ${ }^{* *}$
}

\section{ÖZET}

Amaç: Çalışmamızın amacı, kök kanalı irrigasyonu için farkl irrigasyon solüsyonlarının kullanılmasından sonra gütaperka ile farklı kanal patı kullanılarak yapılan kök kanal dolgusunun dentine ve gütaperkaya bağlanma dayanımının incelenmesidir.

Gereç ve Yöntem: Çalışmamızda 70 adet çekilmiş üst keser ve kanin diş kullanıldı. Dişlerin kök kanal preparasyonları Mtwo (VDW, Munich, Germany) döner sistem eğeler kullanılarak 60.04 nolu eğeye kadar yapıldı. Dişler rastgele olarak her grupta 15 diş olacak şekilde 4 farklı gruba ayrıldı. Ayrıca 5'şer dişten oluşan 2 ayrı kontrol grubu oluşturuldu. Birinci (A) ve Üçüncü (C) gruplarda final irrigasyon olarak $\mathrm{NaOCl}$ ile MTAD solüsyonu sırası ile uygulandı. İkinci (B) ve dördüncü (D) gruplarda ise final irrigasyon olarak $\mathrm{NaOCl}$ ile EDTA uygulandı. Kontrol gruplarında ise irrigasyon solüsyonu olarak sadece $\mathrm{NaOCl}$ kullanıldı. Kök kanal dolguları A ve B gruplarında AH Plus (Dentsply DeTrey, Konstanz, Germany) kanal patı ve gütaperka, $C$ ve $D$ gruplarında ise Endorez (Ultradent, South Jordan, USA) kanal patı ve gütaperka kullanılarak tek kon yöntemiyle yapıldı. Kontrol gruplarından E grubunda kök kanal dolgusu için AH Plus kanal patı ve gütaperka, $\mathrm{F}$ grubunda ise Endorez kanal patı ve gütaperka kullanılarak tamamlandı. Kök kanal dolguları tamamlandıktan sonra dişler nemli ortamda bir hafta kadar bekletildikten sonra dişlerin koronal ve orta üçlü kısmından $3 \mathrm{~mm}$ boyutunda horizontal kesitler alınarak farklı irrigasyon solüsyonu uygulamasının, farklı kanal patlarının dentin duvarına ve gütaperkaya bağlanma kuvvetlerine etkisi push-out yöntemiyle değerlendirildi. Elde edilen verilerin istatistiksel analizleri one way Anova Tukey-Kramer çok yönlü karşılaştırmalı testi kullanılarak yapıldı.

Bulgular: Calışmanın sonucunda elde edilen verilerin ortalama değerleri ve standart sapmaları; MTAD-AH Plus $\left(1.13 \pm 0.18^{\mathrm{a}}\right)$, EDTA-AH Plus $\left(1.10 \pm 0.26^{\mathrm{a}}\right)$, NaOCl-AH Plus $\left(0.77 \pm 0.19^{c}\right)$, MTAD-Endorez $\left(0.99 \pm 0.20^{b}\right)$, EDTA-Endorez $\left(1.02 \pm 0.21^{\mathrm{b}}\right)$ ve $\mathrm{NaOCl}-$ Endorez $\left(0.78 \pm 0.23^{\mathrm{c}}\right) \mathrm{MPa}$ olarak bulundu. İstatistiksel olarak gruplar arasında anlamlı farklılık gözlendi. En yüksek bağlanma direnci MTAD irrigasyon solüsyonu kullanılarak $\mathrm{AH}$ Plus kanal patı ile yapılan kanal dolgusu sonrası elde edildi.

Sonuç: Bu çalışmada, kök kanalının final irrigasyonunda, irrigasyon solüsyonu olarak $\mathrm{NaOCl}$ ile MTAD kullanılmasının ve kanal dolgusu işleminde ise uygun bir gütaperka ile $\mathrm{AH}$ Plus kanal patının kullanılmasının daha etkili olduğu sonucu bulunmuştur.

Anahtar Kelimeler: Endorez, AH Plus, Push-Out.

\section{ABSTRACT}

Aim: The aim of this study was to evaluate the bond strenght of gutta-percha and different root canal sealers to root canal dentine and gutta-percha after using different irrigation solutions.

Method: Seventy extracted superior incisor teeth and canine were used in this study. Root canals were instrumented using Mtwo (VDW, Munich, Germany) rotary system up to $60.04 \mathrm{NiTi}$ file. Teeth were randomly divided in to four groups $(n=15)$ and constituted two control groups $(n=5)$. Both first $(A)$ and third (C) groups, $\mathrm{NaOCl}$ and MTAD were applied as the final irrigation. Both second (B) and fourth (D) groups, $\mathrm{NaOCl}$ and EDTA were applied as final irrigation. In control groups, only $\mathrm{NaOCl}$ was used as irrigation solution. Both groups $\mathrm{A}$ and $\mathrm{C}$ were filled using $\mathrm{AH}$ Plus (Dentsply DeTrey, Konstanz, Germany) and guttapercha single con technique. Both groups $B$ and $D$ were filled using Endorez (Ultradent, South Jordan, USA) and guttapercha with single con technique. First control group was obturated with AH Plus, second control group was obturated with Endorez. Three-millimeter thick horizontal sections from the coronal and mid-thirds of each root were sliced for the push-out bond strength measurement. Statistical analysis was calculated using one-way ANOVA/Tukey type multiple comprasion methods.

Results: The mean values of bond strengths were recorded for different subgroups are presented MTAD-AH Plus $\left(1.13 \pm 0.18^{\mathrm{a}}\right)$, EDTA-AH Plus $\left(1.10 \pm 0.26^{\mathrm{a}}\right)$, NaOCl-AH Plus $\left(0.77 \pm 0.19^{\mathrm{C}}\right)$, MTAD-Endorez $\left(0.99 \pm 0.20^{\mathrm{b}}\right)$, EDTA-Endorez $\left(1.02 \pm 0.21^{b}\right)$ ve NaOCl-Endorez $\left(0.78 \pm 0.23^{c}\right) \mathrm{MPa}$.

Statistically, significant difference was found between the groups. The highest bond strenght is reached after obturation with AH Plus, using MTAD irrigation solution.

Conclusion: According to study, it was concluded that using $\mathrm{NaOCl}$ and MTAD as irrigation solution and obturation using AH-Plus were found to be more effective.

Keywords: Endorez, AH Plus, Push-Out.

\footnotetext{
* Bülen Ecevit Üniversitesi Diş Hekimliği Fakültesi Endodonti Anabilim Dalı, Zonguldak, Türkiye

** Ondokuz Mayıs Üniversitesi Diş Hekimliği Fakültesi Endodonti Anabilim Dalı, Samsun, Türkiye
} 


\section{GİRIŞ}

Endodontik tedavinin başarılı olmasında biyomekanik preparasyon ve sonrasında yapılan etkili ve sızdırmaz bir kanal dolgusu önemli rol oynamaktadır. Mekanik preparasyonda Nikel-titanyum (NiTi) döner eğe sistemlerin kullanılması son zamanlarda oldukça popüler bir yöntem olarak kullanılmakta ve sıklıkla tercih edilmektedir. Bu yöntemin kullanılması ile kök kanal sisteminin kolay, hızlı ve etkili bir şekilde şekillendirilebildiği, ayrıca uygulama sırasında oluşabilecek hatalarının daha az olduğu ve kök kanallarının ana hatlarına daha uygun bir genişletme sağladığı bildirilmiştir. ${ }^{1,2}$ Döner eğe sistemleri 0,02 , 0,04, 0,06 gibi farklı şekil ve açılarda olabilmektedir. ${ }^{2}$ Böylelikle kök kanal sisteminin ana hatlarına uygun bir şekillendirme ve genişletme sağlanırken, apikal genişletmenin minimum seviyede tutularak maksimum apikal sızdırmazlık sağlanabilmektedir. Ayrıca konik formda şekillendirilmesi sağlanan kök kanal sisteminin irrigasyonu daha etkili olmaktadır. ${ }^{3}$

Kök kanal sisteminde dezenfeksiyon ve smear tabakasının uzaklaştırılması amacı ile kliniklerde birçok irrigasyon solüsyonu ve şelasyon ajanı kullanılmaktadır. En yaygın kullanılan irrigasyon solüsyonu $\mathrm{NaOCl}$, en yaygın kullanılan şelasyon ajanı ise EDTA olarak bilinmektedir. ${ }^{2,4}$ Bununla birlikte MTAD içerisinde bulunan asitten dolayı irrigasyon solüsyonu olarak kullanıldığında smear tabakasını da uzaklaştırdığı birçok çalışma ile gösterilmiştir.5-7

Kök kanal sisteminin dezenfeksiyonundan sonra, kök kanalı boyunca tam bir sızdırmazlık sağlamak ve kök kanal tedavisinden sonra tekrar enfekte olmasının engellemek amacı ile obturasyon işlemi yapılmaktadır. 8 Bu amaçla kliniklerde gütaperka ve endodontik patların kombine kullanımı en yaygın ve kabul gören yöntem olmasına rağmen kök kanal sisteminde hermetik bir tıkama sağlamadığı bildirilmiştir. ${ }^{9-11}$ $\mathrm{Bu}$ sorunu gidermek amacı ile farklı birçok teknik ve materyal üretilmiştir. Bu materyallerden Endorez kanal patının kök kanal sisteminde monoblok bir yapı oluşturduğu belirtilmiştir. ${ }^{12,13}$ Endodontik monoblok yapı, kanal dolgu maddesi, patı ve kök kanal dentininin oluşturduğu bütünlük olarak adlandırılmaktadır. ${ }^{12,13}$ Kök kanal patlarının dentine bağlanma dayanımları, kanal dolgusunun örtücülük özellikleri açısından önemli olduğu bilinmektedir. ${ }^{14}$ Kök kanal patlarının bağlanma dayanımlarının ölçülmesinde ise push-out testinin güvenilir olduğu rapor edilmiştir. ${ }^{15,16}$

Bütün bu bilgilerin ışığı altında yapılan bu invitro çalışma ile farklı irrigasyon solüsyonları kullanımının ardından AH Plus-Gütaperka ve EndorezGütaperka kombinasyonu ile yapılan kök kanal dolgusunun dentine bağlanma kuvvetlerinin karşılaştırılarak incelenmesi amaçlanmıştır.

\section{GEREÇ VE YÖNTEM}

Çalışmamızda 70 adet tek köklü üst keser dişler kullanıldı. Dişlerin etrafındaki dokular ve kalsifiye eklentiler periodontal aletler ile uzaklaştırıldı. Dişlerin kron kısımları mine sement hudutundan su soğutmalı bir sistem altında kök boyları yaklaşık $16 \mathrm{~mm}$ olacak şekilde uzaklaştırılarak, giriş kaviteleri açıldı. Daha sonra 15 numaralı K File (Mani Inc., Takanezawa, Japonya) el eğesi ile çalışma boyu tespit edilerek mekanik genişletme işlemi üretici firmanın önerileri doğrultusunda Mtwo (VDW, Munich, Germany) döner sistem eğeler ile 60/.04 nolu eğeye kadar yapıldı. Her eğe değişiminden önce irrigasyon solüsyonu olarak $3 \mathrm{ml} \% 5^{\prime}$ lik sodyum hipoklorit ( $\mathrm{NaOCl}$ ) (Wizard, Rehber Kimya San., İstanbu) kullanıldı. Dişler rastgele olarak her grupta 15 adet diş olacak şekilde 4 farklı gruba ve 5'şer dişten oluşan 2 ayrı kontrol grubuna ayrıldı. A ve $\mathrm{C}$ gruplarında final irrigasyon olarak \% 5 $\mathrm{NaOCl}$ ile \% 5 EDTA (Wizard, Rehber Kimya San., Istanbul) solüsyonu sırası ile uygulandı. B ve D gruplarında ise final irrigasyon olarak \% 1,3 $\mathrm{NaOCl}$ ile MTAD (Dentsply, Tulsa, OK, USA) uyguland. Kontrol gruplarında irrigasyon solüsyonu olarak sadece \% 5 $\mathrm{NaOCl}$ kullanıldı. Kök kanal dolguları $A$ ve $B$ gruplarında AH Plus (Dentsply DeTrey, Konstanz, Germany) kanal patı ve 60/.04 nolu açılı gütaperka ile $C$ ve D gruplarında ise ENDOREZ (Ultradent, South Jordan, USA) kanal patı ve 60/.04 nolu açılı gütaperka kullanılarak tek kon yöntemiyle yapıldı. Kontrol gruplarından birincisinde (E) kök kanal dolgusu için AH-Plus kanal patı ve 60/.04 gütaperka ikincisinde (F) ise Endorez kanal patı ve 60/.04 gütaperka kullanılarak tamamlandı. Kök kanal dolguları tamamlanan dişlerin giriş kaviteleri geçici dolgu maddesi Cavit (3M Espe, Seefeld, Almanya) ile kapatıldı. Dişler nemli bir ortamda bir hafta bekletildikten sonra su soğutmalı bir sistem altında dişlerin koronal ve orta üçlüsünden $3 \mathrm{~mm}$ boyutunda horizontal kesitler alınarak farklı 
irrigasyon solüsyonu uygulamasının kanal patlarının dentin duvarına ve gütaperkaya bağlanma kuvvetlerine etkisi İnstron® (Instron Corp., Canton, MA, U.S.A.) cihazı kullanılarak push-out yöntemiyle ölçüldü (Resim-1,2). ${ }^{15}$

$$
\text { Push-out bond strenght }(\mathrm{MPa})=\frac{\text { maksimum kuwe (Newton) }}{\text { Kanal dolgusunun adheryon alanı }\left(\mathrm{mm}^{2}\right)}
$$

Elde edilen veriler Kolmogorov-Smirnov testine göre verilerin normal dağılış gösterdiği saptandı ve istatistiksel analizinde One-way ANOVA ile $p$ değeri $<0.05$ olduğu gözlendiğinden, Tukey-Kramer çok yönlü karşılaştırmalı testi kullanıldı.

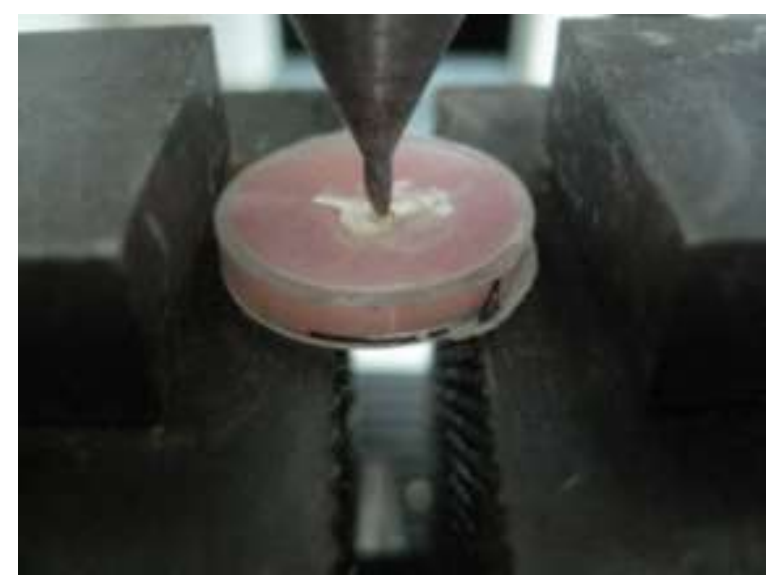

Resim 1. İnstron cihazı ile gütaperka-kanal patına uygulanan kuvvet görüntüsü.

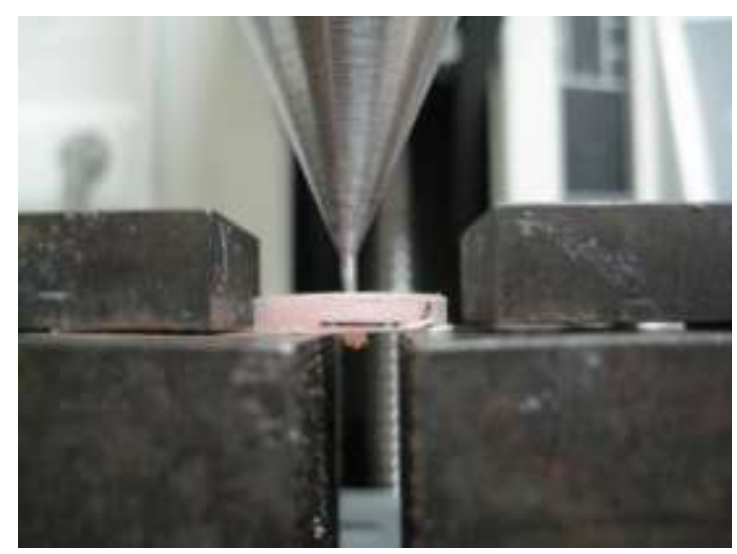

Resim 2. Gütaperkanın kök kanalından ayrıldığı andaki görüntüsü.

\section{BULGULAR}

Elde edilen verilerin istatistiksel sonuçları Tablo $1^{\prime}$ de gösterilmiştir. En yüksek bağlanma direnci MTAD-AH Plus grubunda gözlenirken, en düşük bağlanma direnci kontrol gruplarından $\mathrm{NaOCl}-\mathrm{AH}$ Plus grubunda tespit edilmiştir. A grubu ile B grubu arasında, $C$ grubu ile D grubu arasında ve $E$ grubu ile $F$ grubu arasında istatistiksel olarak farklılık gözlenmezken ( $p>0,05)$, A ve $B$ grubu ile $C$ ve $D$ grupları arasında istatistiksel olarak anlamlı farklılık gözlenmiştir $(p<0,05)$. Benzer şekilde $A, B, C$ ve $D$ grupları ile kontrol grupları arasında da istatistiksel olarak anlamlı bir farklılık olduğu tespit edilmiştir $(p<0,05)$.

Tablo 1. Kullanılan irrigasyon solüsyonları ve kanal patlarına göre elde edilen bağlanma direnci ortalama değerleri ve standart sapmaları (MPa)

\begin{tabular}{|c|c|c|}
\hline \multicolumn{2}{|c|}{ Gruplar } & Mean \pm Sd (MPa) \\
\hline A & EDTA-AH Plus & $1,10 \pm 0,26^{\mathrm{a}}$ \\
\hline B & MTAD-AH Plus & $1,13 \pm 0,18^{\mathrm{a}}$ \\
\hline C & EDTA-Endorez & $1,02 \pm 0,21^{\mathrm{b}}$ \\
\hline D & MTAD-Endorez & $0,99 \pm 0,20^{\mathrm{b}}$ \\
\hline E & NaOCl-AH Plus & $0,77 \pm 0,19^{\mathrm{c}}$ \\
\hline F & NaOCl-Endorez & $0,78 \pm 0,23^{\mathrm{c}}$ \\
\hline
\end{tabular}

*Farklı karakterler arasında istatistiksel olarak anlamlı farklılık gözlenmiştir $(p<0,05)$.

\section{TARTIŞMA}

Başarılı kök kanal tedavisi kök kanal sistemindeki debrisin ve patojenik mikroorganizmaların elimine edilmesine ve sonrasında ağız ortamından ve periapikal dokulardan mikroorganizmaların geçişini engelleyecek sızdırmaz bir kanal dolgusuna bağlı olmaktadır. ${ }^{17}$ Kanal dolgusu olarak kullanılan gütaperkanın tek başına kanal duvarlarına adezyonu olmadığından dolayı kanal patları ile birlikte kullanılması gerekmektedir. Kök kanal patlarının dentine ve gütaperkaya olan bağlanma direnci kök kanal dolgusunun sızdırmazlığı ve bütünlüğü açısından önemli rol oynamaktadır. ${ }^{18}$ Endodontik tedavide çinkooksit öjenol içerikli, cam iyonomer esaslı ve resin esaslı olarak birçok kanal patı kullanılmıştır. Epoksi rezin içerikli kanal patları yıllardır kök kanal dolgu- 
sunda kullanılmakta ve ayrıca birçok çalışmada bu kanal patlarının dentine bağlanma kuvvetinin diğer kanal patlarına göre oldukça yüksek olduğu gösterilmiştir. ${ }^{14,19,20}$ Hidrofilik, üretan dimetakrilat rezin bazlı bir kanal patı olan EndoREZ tek kon tekniği ile kök kanal dolgusu yapılması için geliştirilmiş ve epoksi rezin patlara benzer olarak dentin tübülleri içerisine geniş bir şekilde penetre olabildiği gösterilmiştir. ${ }^{21}$ Kanal patı ile kanal duvarı arasındaki bağlantı ve smear tabakasının bağlanma direnci üzerine olan etkisi birçok çalışma ile incelenmiştir. ${ }^{14,19,20,22-27}$ Kök kanal dolgusu işleminde kullanılan kanal dolgu materyali (gütaperka) ve ince bir tabaka olacak şekilde kullanılan kanal patı ile dentin-kanal patı ve ana kon-kanal patı arasında iki farklı yüzey elde edilmesine rağmen, kök kanalı sadece kanal patı ile doldurulduğunda sadece bir yüzey elde edilmektedir. Endodontik tedavide, kök kanal boşluğu sadece kanal patı ile doldurulmadığından ve klinik açıdan uygun olmayacağı kanısında olduğumuzdan dolayı çalışmamızda kanal patı ve açılı gütaperka (ana kon) ile birilikte yapılan kanal dolgularının dentine bağlanma dirençleri değerlendirilmiştir. Bu araştırmada kök kanal dolgusunun kanaldan ayrıldığı andaki değerler kullanılmıştır. Ancak kanal dolgusunun ayrılmasının önemli olduğu düşünüldüğünden, bu ayrılmanın kanal patı/gütaperka arasındaki bağlantıdan $\mathrm{mı}$ yoksa kanal patı/dentin arasından mı olduğu değerlendirmeye alınmamıştır. Bu nedenle konu hakkındaki eksikliklerin bu yöndeki yapılan çalışmalar ile giderilebileceği kanısındayız. Bağlanma direncinin değerlendirilmesinde birçok yöntem kullanılmaktadır. ${ }^{15,25,28-31}$ Ancak çalışmamızda güncel ve rasyonel değerler vermesinden dolayı push-out testi kullanılmıştır.

Jainaen ve arkadaşlarının ${ }^{32}$ yapmış oldukları çalışmalarında $\mathrm{AH}$ Plus ve ana kon kombinasyonun Endorez ana kon kombinasyonundan daha üstün olduğunu tespit etmişlerdir. Ayrıca Endorez için elde edilen değeri $(0,9 \mathrm{MPa})$ çalışmamızda elde edilen değerlere $(1,02$ ve $0,99 \mathrm{MPa})$ oldukça benzerlik göstermektedir. Nagas ve arkadaşları ${ }^{33}$ da farklı kanal patlarının ana kon ile birlikte kullanılmaları ve bağlanma dirençlerini değerlendirdikleri çalışmalarında da benzer şekilde $\mathrm{AH}$ Plus ve ana kon kombinasyonun Endorez ve ana kon kombinasyonundan üstün olduğunu ayrıca Endorez-ana kon kombinasyonu ile elde etikleri bağlanma direnci değerlerinin $(1,3,1,0$ ve 0,7 MPa) çalışmamızdaki değerler (1,02 ve 0,99 MPa) ile oldukça uyumluluk gösterdiği tespit edilmiştir. Patil ve arkadaşları ${ }^{34}$ EndoRez ile AH Plus kanal patı ve gütaperka kullanarak yapmış oldukları çalışmalarında, irrigasyon solüsyonu olarak $\mathrm{NaOCl}$ ve EDTA kullanmışlardır. Kök kanal dolguları yapılan dişlerden $2 \mathrm{~mm}$ 'lik horizontal kesitler alarak push-out yöntemi ile kanal patlarının bağlanma dayanımlarını değerlendirmişlerdir. Elde ettikleri sonuçlar AH Plus gütaperka kombinasyonu için 1,49 MPa, EndoRez için bu değerin 0,56 MPa olduğunu bulmuşlardır. Çalışmamızda elde edilen sonuçların önceki çalışmalarla oldukça benzerlik göstermesine rağmen aradaki farklıı̆ı̆ın nedeni olarak kullanılan solüsyonların, kök kanal dolgusu için kullanılan gütaperka konlarının açılarının farklı olmasından ve horizontal olarak elde edilen kesit kalınlıklarının farklı olmasından kaynaklandığı kanısındayız. Ayrıca önceden yapılan çalışmaların birçoğunda irrigasyon solüsyonu olarak sadece $\mathrm{NaOCl}$ ve EDTA kullanılmıştır. $\mathrm{Bu}$ çalışmada ise MTAD irrigasyon solüsyonu kullanılması ve sonrasında farklı materyaller kullanılarak yapılan kök kanal dolgusunun dentine bağlanma direnci ile ilgili literatürde çok az sayıda çalışma bulunmaktadır. ${ }^{35,36} \mathrm{Bu}$ yüzden yapılan bu çalışma ile literatüre katkı sağlanmaya çalışılmıştır.

Sonuç olarak kanal patlarının dentine adhezyonunda smear tabakasının kaldırımasının öneminin büyük olduğu ve $\mathrm{AH}$ Plus-Gütaperka kombinasyonunun bağlanma direncinin Endorez kök kanal dolgu sisteminden üstün olduğu gözlenmiştir.

\section{KAYNAKLAR}

1. Tan BT, Messer HH. The quality of apical canal preparation using hand and rotary instruments with specific criteria for enlargement based on initial apical file size. J Endod 2002;28:658-64.

2. Rudddle $\mathrm{CJ}$. Cleaning and shaping the root canal system. In: Cohen S, Burns R, editors. Pathways of the pulp. 10th ed. St Louis (MO): Mosby; 2010. p. 231-92.

3. Coldero LG, McHugh S, MacKenzie D, Saunders WP. Reduc-tion in intracanal bacteria during root canal preparation with and without apical enlargement. Int Endod J 2002;35:437-46.

4. Evcil MS, Ersoy İ, Yeter Yeşildal K, Topcu Colak M. A comparison of the accuracy of two different apex locators usıng different root canal solutıons. Atatürk Üniv Diş Hek Fak Derg 2012;22:132-7. 
5. Torabinejad M, Johnson WB (Inventors, Assignee): Irrigation solution and methods for use, US Patent and Trademark Office, December 25, 2003.

6. Torabinejad M, Khademi AA, Babagoli J, Cho Y, Johnson WB, Bozhilov $K$, et al. A new solution for the removal of the smear layer. J Endod 2003;29:170-5.

7. Torabinejad $M$, Shabahang $S$, Aprecio RM, Kettering JD. The antimicrobial effect of MTAD: an invitro investigation. J Endod 2003;29:400-3.

8. Stoll $R$, Betke $K$, Stachniss V. The influence of different factors on the survival of root canal fillings: a 10-year retrospective study. J Endod 2005;31:783-90.

9. Dummer PM. Comparison of undergraduate endodontic teaching programmes in the United Kingdom and in some dental schools in Europe and the United States. Int Endod J 1991;24:169-77.

10. Shipper G, Ørstavik D, Teixeira FB, Trope M. An evaluation of microbial leakage in roots filled with a thermoplastic synthetic polymer-based root canal filling material (Resilon). J Endod 2004;30:342-7.

11. Shipper G, Teixeira FB, Arnold RR, Trope M. Periapical inflammation after coronal microbial inoculation of dog roots filled with gutta-percha or resilon J Endod 2005;31:91-6.

12. Tay FR, Pashley DH. Monoblocks in root canals: a hypothetical or a tangible goal. J Endod 2007;33:391-8. Review.

13. Gillespie WT, Loushine RJ, Weller RN, Mazzoni A, Doyle MD, Waller JL, Pashley DH, Tay FR. Improving the performance of EndoREZ root canal sealer with a dual-cured two-step self-etch adhesive. II. Apical and coronal seal. J Endod 2006;32:771-5.

14. Tagger $M$, Tagger $E$, Tjan AH, Bakland LK. Measurement of adhesion of endodontic sealers to dentin. J Endod 2002;28:351-4.

15. Nagas E., Cehreli ZC, Durmaz V, Vallittu PK, Lassila LV. Regional push-out bond strength and coronal microleakage of Resilon after different light-curing methods. J Endod 2007;33:1464-8

16. Goracci C, Tavares AU, Fabianelli A, Monticelli F, Raffaelli O, Cardoso PC, Tay F, Ferrari M. The adhesion between fiber posts and root canal walls: comparison between microtensile and push-out bond strength measurements. Eur J Oral Sci 2004;112:353-61.
17. Sundqvist G, Figdor D, Persson S, Sjogren U. Microbiologic analysis of teeth with failed endodontic treatment and the outcome of conservative re-treatment. Oral Surg, Oral Med, Oral Pathol, Oral Radiol, Oral Endod 1998;85:8693.

18. Skinner RL, Himel VT. The sealing ability of injection-molded thermoplasticized gutta-percha with and withoutthe use of sealers. J Endod 1987;13:315-7.

19. Wennberg A, Ørstavik D. Adhesion of root canal sealers to bovine dentine and gutta-percha. Int Endod J 1990;23:13-9.

20. Lee KW, Williams MC, Camps JJ, Pashley DH. Adhesion of endodontic sealers to dentin and gutta-percha. J Endod 2002;28:684-8.

21. Bergmans L, Moisiadis P, De Munck J, Van Meerbeek B, Lambrechts P. Effect of polymerization shrinkage on the sealing capacity of resin fillers for endodontic use. J Adhes Dent 2005;7:321-9.

22. Saleh IM, Ruyter IE, Haapasalo MP, Ørstavik D. Adhesion of endodontic sealers: scanning electron micro-scopy and energy dispersive spectroscopy. J Endod 2003;29:595-601.

23. Gogos C, Economides N, Stavrianos C, Kolokouris I, Kokorikos I. Adhesion of a new methacrylate resin-based sealer to human dentin. Journal of Endodontics 2004;30:238-40.

24. Doyle MD, Loushine RJ, Agee KA et al. Improving the performance of EndoRez root canal sealer with a dual-cured two-step self-etch adhesive. I. Adhesive strength to dentin. J Endod 2006;32:766-70.

25. Eldeniz AU, Erdemir A, Belli S. Shear bond strength of three resin based sealers to dentin with and without the smear layer. J Endod 2005;31:293-6.

26. Hayashi M, Takahashi Y, Hirai M, Iwami Y, Imazato $\mathrm{S}$, Ebisu S. Effect of endodontic irrigation on bonding of resin cement to radicular dentin. Eur J Oral Sci 2005;113:70-6.

27. Saleh IM, Ruyter IE, Haapasalo M, Ørstavik D. The effects of dentine pretreatment on the adhesion of root-canal sealers. Int Endod J 2002;35:859-66.

28. Barbizam JV, Trope M, Tanomaru-Filho M, Teixeira $\mathrm{EC}$, Teixeira FB. Bond strength of different endodontic sealers to dentin: push-out test. J Appl Oral Sci. 2011;19:644-7. 
29. Fisher MA, Berzins DW, Bahcall JK. An in vitro comparison of bond strength of various obturation materials to root canal dentin using a push-out test design. J Endod. 2007;33:856-8.

30. Teixeira CS, Alfredo E, Thomé LH, Gariba-Silva R, Silva-Sousa YC, Sousa-Neto MD. Adhesion of an endodontic sealer to dentin and gutta-percha: shear and push-out bond strength measurements and SEM analysis. J Appl Oral Sci. 2009;17:129-35.

31. Gesi A, Raffaelli O, Goracci C, Pashley DH, Tay FR, Ferrari M. Interfacial strength of resilon and guttapercha to intraradicular dentin. J Endod. 2005;31:809-13.

32. Jainaen A, Palamara JEA, Messer HH. Push-out bond strengths of the dentine-sealer interface with and without a main cone. Int Endod J 2007; 40:882-90.

33. Nagas E, Altundasar E, Serper A. The effect of master point taper on bond strenght and apical sealing ability of different root canal sealers. Oral Surg Oral Med Oral Pathol Oral Radiol Endod 2009;107:61-4.

34. Patil SA, Dodwad PK, Patil AA. An in vitro comparison of bond strengths of Gutta-percha/AH Plus, Resilon/Epiphany self-etch and EndoREZ obturation system to intraradicular dentin using a push-out test design. J Conserv Dent. 2013;16:238-42.

35. Hashem AA, Ghoneim AG, Lutfy RA, Fouda MY. The effect of different irrigating solutions on bond strength of two root canal-filling systems. J Endod 2009;35:537-40.

36. Shokouhinejad N, Sharifian MR, Jafari M, Sabeti MA. Push-out bond strength of Resilon/Epiphany self-etch and gutta-percha/AH26 after different irrigation protocols. Oral Surg Oral Med Oral Pathol Oral Radiol Endod 2010;110:88-92.

\section{Yazışma Adresi:}

Dr. Dt. Ersan ÇIÇEK

Bülen Ecevit Üniversitesi

Diş Hekimliği Fakültesi

Endodonti Anabilim Dalı, Zonguldak

Tel: 0372-261-3427

e-posta:ersancicek@gmail.com 